\title{
Industrie- und regionalpolitische Entwicklungsmuster in Europa
}

Trotz europäischer Strukturpolitik bestehen die ökonomischen und sozialen Unterschiede innerhalb der Europäischen Union (EU) weiter. Dies ist unter anderem eine Folge ihrer Erweiterung und der Globalisierung. Zudem fehlt das Pendant zur Wirtschafts- und Währungsunion, die Sozialunion. Hier besteht dringender Nachholbedarf, um soziale Unterschiede und Spannungen zu vermindern. Innerhalb des EU-Maßnahmenpakets ist die Gratwanderung zwischen Wachstums- und Ausgleichszielsetzung ,auszuhalten“. Nicht nur wegen des Kohäsionsziels wäre eine Verschiebung der Akzente zugunsten einer Förderung von Exzellenz und Wachstum fatal.Vielmehr ist eine gute Kohäsionspolitik eine zentrale Voraussetzung für die Stimulierung von Wachstumspotenzialen.

\section{Eine veränderte Ausgangslage}

Die Öffnung der Märkte für Produkte und Dienstleistungen, die Beseitigung der Hemmnisse für den Transfer von Kapital und Arbeitsleistungen, die Einführung einer einheitlichen Währung sowie die Osterweiterung prägen den aktuellen Entwicklungsprozess der Europäischen Union. Mit der Erweiterung und Vertiefung der EU haben sich die gesamtwirtschaftlichen Rahmenbedingungen für die Entwicklung der Branchen und Regionen in Europa nachhaltig verändert. Trotz eines einheitlichen Rahmens bestehen Unterschiede in den Arbeits- und Lebensbedingungen sowie in den Entwicklungsperspektiven von Branchen und Regionen fort. Denn eine völlige Angleichung, z. B. der Steuer- und Abgabensysteme, oder der sozialen Sicherungssysteme, ist noch lange nicht in Sicht und möglicherweise politisch auch nicht gewollt. Der veränderte gesamtwirtschaftliche Rahmen ist für sich genommen schon so umfassend, dass tief greifende Strukturveränderungen damit verbunden waren und sind.

Zugleich fördert die Globalisierung Differenzierungsprozesse in Europa. Manche Regionen und Branchen können die Anforderungen einer zunehmenden Internationalisierung der Wirtschaftsbeziehungen, der Bildung neuer Wertschöpfungsketten sowie eines wachsenden Einflusses der Kapitalmärkte bewältigen. Andere jedoch nicht. Im Zuge der Verkürzung der Produkt-Lebenszyklen steigt der Innovationsdruck, dem viele alte Zentren von Pro- duktion und Dienstleistungen nicht gewachsen sind. Einige hingegen konnten ihre Position verstärken. Im Ergebnis jedoch führt diese Entwicklung nicht zu einer zunehmenden Kohäsion, sondern eher zu einer Ausdifferenzierung in den ökonomischen Potenzialen von Branchen und Regionen - mit den entsprechenden Auswirkungen auf die Lebenslagen der Bevölkerung. Zudem verstärken sich die Differenzierungsprozesse zumindest auf mittlere Sicht durch den EU-Beitritt von Ländern mit einem deutlichen wirtschaftlichen Entwicklungsrückstand.

Ziel des Beitrags ist, zum einen die Auswirkungen dieses hier grob skizzierten Prozesses für die Branchen- und Regionalentwicklung in Europa aufzuzeigen. Zum anderen soll diskutiert werden, welche Rolle die Politik dabei spielt bzw. spielen will.

\section{Wirtschaftliche Entwick- lungsmuster in Europa}

\subsection{BRANCHENSTRUKTUREN}

Nach wie vor bestehen deutliche Unterschiede in den Branchenstrukturen der EU-Mitgliedsländer (Tabelle 1). Deutschland ist durch einen vergleichsweise großen industriell-gewerblichen Bereich gekennzeichnet. Abgesehen von der Mehrzahl der mittel- und osteuropäischen Mitgliedsländer hat kein anderes Land der EU-15 absolut wie auch relativ so viele Beschäftigte im Produzierenden Gewerbe. Dementsprechend niedrig fällt in Deutschland der Beschäftigtenanteil in den Dienstleistungsbereichen aus. Insbesondere gibt es einen
Rückstand bei den wirtschaftsnahen Dienstleistungen, der auf statistische Defizite bei den industrienahen bzw. unternehmensbezogenen Dienstleistungen verweist. Offensichtlich neigen deutsche Unternehmen trotz massiver Auslagerungs- und Umstrukturierungsprozesse in jüngster Zeit dazu, Dienstleistungen selbst zu erstellen, was statistisch den industriellen Branchen zugerechnet wird. Gemessen am deutschen und europäischen Durchschnitt sind vor allem die französische und britische Wirtschaft durch einen hohen Dienstleistungsbereich geprägt.

Insgesamt zeigt der Vergleich, dass die EU-Länder noch recht unterschiedlich strukturiert und in einzelnen Ländern jeweils typische Branchenschwerpunkte $\mathrm{zu}$ finden sind. Dies gilt vor allem für die kleinen europäischen Länder, wie z. B. Luxemburg, Belgien oder Dänemark, mit ausgeprägter Spezialisierung, z. B. bei den Banken oder bei staatlichen Institutionen. Ähnliches gilt auch für die Landwirtschaft und die Verbrauchsgüterindustrie in den süd- und osteuropäischen Ländern.

Diese Strukturunterschiede scheinen sich in den letzten Jahren noch verfestigt zu haben. So blieb z. B. das Beschäftigten-

Frank Gerlach, Dr., Leiter des Referats "Strukturwandel - Innovationen und Beschäftigung " in der Forschungsförderung der Hans-Böckler-Stiftung. e-mail: Frank-Gerlach@boeckler.de Astrid Ziegler, Dr., ist Wissenschaftlerin im WSI in der Hans-Böckler-Stiftung. Arbeitsschwerpunkte: Strukturforschung und -politik.

e-mail: Astrid-Ziegler@boeckler.de 
Tabelle 1: Beschäftigte in ausgewählten Ländern der EU-25 nach Wirtschaftsbereichen* 2005 - in \% -

\begin{tabular}{|c|c|c|c|c|c|c|c|c|c|c|c|c|}
\hline & Belgien & $\begin{array}{l}\text { Däne- } \\
\text { mark }\end{array}$ & $\begin{array}{l}\text { Deutsch- } \\
\text { land }\end{array}$ & Spanien & $\begin{array}{l}\text { Frank- } \\
\text { reich }\end{array}$ & Italien & $\begin{array}{l}\text { Luxem- } \\
\text { burg }\end{array}$ & Portugal & $\begin{array}{c}\text { Groß- } \\
\text { britannien }\end{array}$ & $\begin{array}{l}\text { Tschech. } \\
\text { Republik }\end{array}$ & Ungarn & Litauen \\
\hline Land- und Forstwirtschaft, Fischerei & 2,42 & 3,48 & 3,09 & 5,52 & 4,64 & 4,43 & 1,94 & 12,23 & 1,37 & 4,17 & 5,00 & 17,68 \\
\hline $\begin{array}{l}\text { Produzierendes Gewerbe } \\
\text { davon: }\end{array}$ & 19,42 & 21,58 & 30,86 & 30,53 & 24,80 & 28,82 & 18,02 & 30,99 & 21,74 & 38,48 & 31,42 & 31,41 \\
\hline Nahrungs- und Genussmittel $(15+16)$ & 2,82 & 0,36 & 3,32 & 2,68 & 0,35 & 0,22 & 1,44 & 2,18 & 1,39 & 2,68 & 3,75 & 4,04 \\
\hline Textil, Leder und Bekleidung (17-19) & 1,11 & 0,34 & 0,87 & 1,18 & 0,91 & 3,12 & 0,27 & 5,89 & 0,41 & 2,23 & 2,58 & 4,78 \\
\hline $\begin{array}{l}\text { Herstellung von chemischen } \\
\text { Erzeugnissen (24) }\end{array}$ & 2,40 & 1,49 & 2,13 & 0,97 & 0,15 & 1,14 & 0,23 & 0,51 & 0,95 & 0,98 & 1,23 & 0,21 \\
\hline Metallerzeugnisse $(27+28)$ & 3,13 & 1,81 & 4,56 & 2,64 & 2,84 & 3,67 & 2,66 & 2,21 & 1,64 & 5,22 & 2,81 & 1,68 \\
\hline Maschinenbau (29) & 1,35 & 2,60 & 3,95 & 1,35 & 1,64 & 3,06 & 0,51 & 0,90 & 1,36 & 2,92 & 1,63 & 0,59 \\
\hline Rundfunk- und Nachrichtentechnik (32) & 0,47 & 0,32 & 0,93 & 0,21 & 0,65 & 0,46 & 0,00 & 0,26 & 0,35 & 0,72 & 1,93 & 0,66 \\
\hline $\begin{array}{l}\text { Medizin-, Mess-, Steuer- und } \\
\text { Regelungstechnik, Optik, } \\
\text { Herstellung von Uhren (33) }\end{array}$ & 0,25 & 0,69 & 1,06 & 0,17 & 0,70 & 0,58 & 0,55 & 0,14 & 0,04 & 0,51 & 0,00 & 0,24 \\
\hline Fahrzeuge $(34+35)$ & 1,91 & 0,07 & 4,41 & 1,69 & 2,43 & 1,61 & 0,05 & 0,36 & 1,66 & 2,18 & 1,50 & 0,61 \\
\hline Baugewerbe (45) & 0,74 & 7,80 & 0,89 & 13,08 & 8,04 & 9,29 & 8,82 & 11,12 & 7,93 & 9,90 & 8,39 & 10,02 \\
\hline $\begin{array}{l}\text { Handel, Verkehr, Dienstleistungen } \\
\text { davon: }\end{array}$ & 78,16 & 74,94 & 66,04 & 63,95 & 70,55 & 66,76 & 80,04 & 56,78 & 76,88 & 57,35 & 63,58 & 50,90 \\
\hline Handel (50-52) & 13,27 & 15,81 & 5,54 & 12,52 & 14,50 & 16,39 & 4,65 & 15,91 & 15,40 & 13,13 & 15,78 & 15,90 \\
\hline Gastgewerbe (55) & 3,93 & 2,34 & 4,79 & 7,46 & 3,91 & 5,25 & 4,24 & 5,45 & 4,30 & 3,95 & 4,28 & 2,52 \\
\hline Kreditwesen und Versicherungen $(65+67)$ & 4,23 & 3,22 & 4,66 & 2,61 & 3,55 & 0,30 & 11,05 & 1,96 & 4,24 & 2,07 & 0,83 & 0,77 \\
\hline Wirtschaftsnahe Dienstleistungen (70-74) & 8,25 & 8,07 & 3,03 & 9,36 & 12,01 & 9,96 & 8,94 & 5,87 & 11,58 & 6,01 & 7,44 & 4,14 \\
\hline Öffentliche Dienstleistungen (75+80) & 22,12 & 14,50 & 17,72 & 12,86 & 9,77 & 14,48 & 21,97 & 13,41 & 16,32 & 13,42 & 16,31 & 6,55 \\
\hline $\begin{array}{l}\text { Gesundheits-, Veterinär- und } \\
\text { Sozialwesen (85) }\end{array}$ & 14,03 & 19,32 & 14,42 & 6,27 & 14,85 & 7,30 & 10,88 & 6,51 & 12,43 & 7,02 & 6,97 & 8,74 \\
\hline Sonstige Dienstleistungen (90-99) & 5,70 & 5,96 & 8,80 & 8,20 & 7,21 & 7,10 & 10,77 & 4,93 & 5,65 & 4,16 & 4,49 & 5,82 \\
\hline
\end{tabular}

wachstum im Bereich Handel, Verkehr und Dienstleistungen in Deutschland hinter dem EU-Durchschnitt zurück. Der fast durchgängige Rückzug der europäischen Textil- und Bekleidungsindustrie traf Italien bislang am wenigsten.

Generell scheinen die europäischen Volkswirtschaften damit eher von einer Differenzierung und Spezialisierung als einer Angleichung - einer strukturellen Konvergenz - bei der Branchenentwicklung geprägt zu sein. Die Harmonisierung des rechtlichen und institutionellen Rahmens in der EU hat nicht zu einer eindeutig erkennbaren Konvergenz der Wirtschaftsstrukturen geführt. Die Unterschiede in den Standortbedingungen erfordern wohl einen langfristigen Anpassungsprozess, sodass die sektorale Wirtschaftsstruktur in der EU noch eine geraume Zeit nationale Prägungen zeigen wird.

\subsection{INNOVATIONSUNTERSCHIEDE}

Wenn Europa gemäß der Lissabon-Strategie eines der wachstumsstärksten Gebiete in der Welt werden soll, dann erfordert dies ein hohes $\mathrm{Ma} ß$ an Innovationsfähigkeit. Von einer konsequenten Nutzung der Innovationspotenziale kann jedoch keine Re- de sein, wenn man die Unterschiede in den Forschungs- und Entwicklungsaktivitäten betrachtet.

Als Indikator verwenden wir die Forschungs- und Entwicklungsintensität, das heißt, den Anteil der Brutto-Inlandsaufwendungen für Forschung und Entwicklung (FuE) in Prozent des Bruttoinlandsproduktes. Im Länder- bzw. Regionenvergleich kann er wichtige Hinweise auf zukünftige und differierende Wachstumspotenziale geben. ${ }^{1}$

Länder wie Schweden oder Finnland „glänzen“ mit einem hohen Anteil an FuEAusgaben von über $4 \%$ bzw. etwa 3,5\% und haben diesen Anteil in den letzten Jahren noch steigern können (OECD 2004). Deutschland bewegt sich mit etwa 2,5\% in der Spitzengruppe, konnte aber seine Intensität - abgesehen von zyklischen Veränderungen - in den letzten 20 Jahren nicht steigern. Europas „Problemkinder“ sind jedoch Länder wie Polen oder auch Italien, mit einem Anteil von unter bzw. 1 \%. Solche Länder bieten naturgemäß jungen Nachwuchsforschern in staatlichen Forschungseinrichtungen, aber auch in der Wirtschaft, deutlich weniger Perspektiven, als sie ihre Altersgenossen etwa in skandinavischen Ländern haben.
Im Durchschnitt gibt die europäische Union 1,9\% des Bruttoinlandsproduktes für Forschung und Entwicklung aus. Hingegen bringt es Japan auf ca. $3 \%$, und die Schwellenländer, das heißt, Staaten wie China, Korea, Indien, Singapur haben ihren Anteil innerhalb von knapp zehn Jahren von $0,9 \%$ auf $1,7 \%$ steigern können. Als Schlaglicht nur eine Zahl, in welcher Weise sich die Strukturen in den letzten Jahren fulminant verändert haben: China

\footnotetext{
1 Zur Bewertung der technologischen Leistungsfähigkeit bzw. der Innovationsfähigkeit einer nationalen Ökonomie, Industrie oder von Dienstleistungen (Schmidt 2006) müssten weitere Indikatoren hinzugezogen werden. Technologien können von außen übernommen werden. Zudem umfasst ein systemisch orientiertes, nicht technikzentriertes Innovationsverständnis wesentlich mehr Elemente, als sie durch den Indikator FuE-Intensität abgebildet werden (Lundvall 1992). Die Innovationsfähigkeit und die internationale Wettbewerbsfähigkeit einer Volkswirtschaft werden durch spezifische Wirtschaftsstrukturen (mit)bestimmt, die leistungsfähige Industrie- und Dienstleistungen, Wertschöpfungsketten und Clusterstrukturen umfassen. Zudem findet Innovation in vielen kleinen und mittleren Unternehmen statt - ohne sich in den FuE-Aufwendungen niederzuschlagen. Dieses gilt es, im Folgenden zu berücksichtigen.
} 
hat im Jahre 2006 mehr als 130 Mrd. Dollar für Forschung und Entwicklung ausgegeben und liegt damit, in absoluten Zahlen gemessen, weltweit nach den USA an zweiter Stelle.

Bestätigt wird unser Befund einer starken Differenzierung innerhalb der EU durch den Innovationsindikator des Deutschen Instituts für Wirtschaftsforschung (DIW). Dieser ist sehr breit angelegt und versucht, mit vielen Einzelindikatoren, die sieben Bereichen zugeordnet werden (Forschung und Entwicklung, Bildung, Finanzierung von Innovationen, Vernetzung der Innovationsakteure, Umsetzung von Innovationen am Markt, innovationsfördernde Regulierung und Wettbewerb, innovationsfreundliche Nachfrage), die Innovationsfähigkeit zu erfassen (Belitz et al. 2006). Danach ergeben sich deutliche Differenzen, wobei wiederum die skandinavischen Länder führend sind, Deutschland sich im Mittelfeld platziert und Spanien und Italien weit abgeschlagen das untere Ende markieren.

\subsection{REGIONALE ENTWICKLUNGS- MUSTER}

In den letzten Jahren ist die regionale Entwicklung in Europa durch die eingangs skizzierten endogenen Faktoren zunehmend in Fluss geraten. Zwar betreibt die Europäische Union schon seit den 1970er Jahren eine Regionalpolitik; jedoch erst seit der grundsätzlichen Reform der Europäischen Strukturfonds Ende der 1980er Jahre und den verschiedenen Erweiterungsrunden, die die regionalen Unterschiede zwischen den Mitgliedstaaten und viel stärker noch zwischen den europäischen Regionen vergrößert haben, sind bestimmte Probleme bewusst geworden. Seither wurde regionalpolitisch darüber diskutiert, ob die europäische Kohäsionspolitik bestehende Diskrepanzen zwischen den Regionen in der Tendenz nivelliert (Konvergenzhypothese), oder ob diese den wirtschaftsstarken Regionen nutzen und damit Unterschiede eher verstärken (Divergenzhypothese).

Die im Jahr 2004 vollzogene Erweiterung war die bislang größte und besonders in ökonomischer und sozialer Hinsicht anspruchsvollste Runde. Denn in ihrem Zuge vergrößerte sich die EU-15 um $34 \%$, und die Bevölkerung wuchs im Vergleich zum Stand von 2000 um $28 \%$. Auch das Bruttoinlandsprodukt der EU wurde um $5 \%$ erhöht, was aber - angesichts der Größe und Anzahl der Beitrittsländer - im Umkehrschluss auch bedeutet, dass sich das durchschnittliche EU-Bruttoinlandsprodukt um $18 \%$ verringerte. Anders als in den Erweiterungsrunden vergangener Jahre sind in einer Phase mit schwachem Wirtschaftswachstum und massiven Beschäftigungsproblemen innerhalb der EU ihr zehn Staaten neu beigetreten, deren Bruttoinlandsprodukt zum Teil unterhalb von $50 \%$ des EU-Durchschnitts lag, und die auch nach dem offiziellen Beitritt im Mai 2004 noch erhebliche Transformationsprobleme zu bewältigen hatten und bis heute haben. Durch den EU-Beitritt von Rumänien und Bulgarien im Januar 2007 sind zwei weitere Länder mit erheblichem wirtschaftlichen Entwicklungsrückstand in die EU gekommen.

In regionaler Hinsicht haben die Einkommens- und Beschäftigungsunterschiede innerhalb der EU mit den letzten beiden Erweiterungsrunden deutlich zugenommen. Nach Berechnungen der Europäischen Kommission (2006) erwirtschafteten $200210 \%$ der Bevölkerung in der EU-27, die in den wohlhabendsten Regionen lebten, über $19 \%$ des gesamten BIP der EU-27, während auf die $10 \%$ der Bevölkerung in den weniger wohlhabenden Regionen nur $1,5 \%$ des BIP entfielen. In Kaufkraftstandards (KKS) ${ }^{2}$ umgerechnet, bewegten sich die Einkommensunterschiede zwischen $15 \%$ in den reichsten Regionen und $3 \%$ des BIP in KKS in den ärmsten Regionen. In KKS betrug das Verhältnis des jeweiligen Anteils am BIP zwischen den $10 \%$ der Bevölkerung in den wohlhabendsten Regionen und den 10 \% der Bevölkerung in den weniger wohlhabenden Regionen $5: 1$ - ohne die KKS-Anpassung $12,5: 1$. Insgesamt 100 Regionen einschließlich 16 Regionen mit Phasing-outStatus fallen unter $75 \%$ des durchschnittlichen Pro-Kopf-Einkommens. Diese Einkommensschwelle entspricht der alten und neuen Grenze, um im Rahmen der Europäischen Strukturfonds als Konvergenzregion eingestuft zu werden und die höchste Förderpriorität zu erhalten.

Doch in den ärmsten Mitgliedstaaten der EU ist die Wirtschaft in den letzten Jahren am schnellsten gewachsen. So weisen die Baltischen Staaten, die Slowakei, aber auch Griechenland, Irland sowie Rumänien und Bulgarien Wachstumsraten von 3,6 \% und mehr auf. Demgegenüber lagen die entsprechenden Zahlen in den „alten“
EU-Staaten bei 1,5 bis $2 \%$. Bis aber Polen (5.000 € BIP pro Einwohner) das deutsche Wohlstandsniveau mit über $26.000 €$ je Einwohner tatsächlich erreicht, ist es noch ein langer Weg. Nach Berechnungen der Europäischen Kommission wird dieser Prozess bei zugrunde gelegten Wachstumsraten von $2 \%$ für die alten Mitgliedsländer und $4 \%$ für die neuen Länder bis zum Jahr 2040 dauern (European Commission 2006).

\subsection{KONZENTRATION AUF STÄDTE}

Analysen zur Entwicklung der Spitzenregionen zeigen hingegen, dass es sich bei diesen in der Regel um Zentren von nationaler, wenn nicht sogar von globaler Bedeutung handelt,

- die im sekundären Sektor durch einen ausgeprägten Besatz mit Headquarterfunktionen gekennzeichnet sind;

- die ein weites Spektrum an unternehmensorientierten Dienstleistungen aufweisen, die sich positiv auf Unternehmensentscheidungen auswirken;

- die häufig Standorte von Ministerien und Behörden sind und so großen Einfluss auf die politische und ökonomische Entwicklung eines Landes ausüben und

- die über eine gute bis sehr gute Erreichbarkeit durch eine internationale und interkontinentale Anbindung in Form von Flughäfen, Schnellbahn- und Autobahnverbindungen sowie eine hervorragende Telekommunikationsinfrastruktur verfügen.

$\mathrm{Zu}$ dieser Gruppe gehören Städte wie London, Paris, Barcelona, München und Frankfurt. Allerdings interpretiert die Fachwelt historisch gesehen leichte Nachteile für Deutschland. Auf Grund des stark zentralistischen Staatsaufbaus entwickelten sich z. B. die Hauptstädte in Frankreich und England schon frühzeitig zu großen Ballungsräumen, wie Paris oder London zeigen. In Deutschland hingegen hat sich ein föderalistischer Staat entwickelt, geprägt

Kaufkraftstandards sind eine statistische Maßeinheit, die die Unterschiede zwischen den nationalen Preisniveaus widerspiegelt. Mit KKS umgerechnete Wirtschaftsindikatoren - das entsprechende Aggregat in laufenden Preisen und nationaler Währung wird durch die entsprechende Kaufkraftparität geteilt - lassen aussagekräftige Vergleiche zwischen den Mitgliedsländern zu. 
durch eine polyzentrale Struktur. Es findet sich keine einzelne großstädtische Agglomeration, die alle anderen weit überragt, sondern eine Anzahl ähnlich entwickelter Großstadtregionen mit metropolitanen Strukturen. Auch viele nichtmetropolitane Räume sind in Deutschland arbeitsteilig mit den dynamischen Metropolräumen verflochten.

Daneben ist generell eine Renaissance der Städte im europäischen Kontext zu beobachten. Sie entwickelten sich in den letzten Jahren zu Zentren des Produzierenden Gewerbes oder spezialisieren sich auf Dienstleistungsbereiche wie z. B. den Fremdenverkehr. Im Vergleich zu den Ballungsräumen besitzen sie allerdings eine geringere Ausstrahlungskraft auf das Umland mit der Folge, dass sich geografische Inseln mit einem relativ hohen Arbeitsproduktivitätsniveau gemessen am nationalen Durchschnitt ergeben. Die Rolle der Städte und Metropolregionen hat in den traditionellen Konzepten der Politik bisher keinen Niederschlag gefunden. Dies hat sich mit dem neuen Konzept der Europäischen Strukturfonds verändert. Denn die nachhaltige Städtepolitik ist als ein wichtiger Förderbereich im neuen Konzept der Europäischen Strukturfonds ab 2007 aufgenommen worden. Auch im Zusammenhang mit der Weiterentwicklung der europäischen Raumentwicklungspolitik wird über spezielle Fördermöglichkeiten nachgedacht. Die Leitbildvorstellung geht in zwei Richtungen: die Raumordnungspolitik soll zukünftig das gesamtwirtschaftliche Wachstum fördern, indem einerseits räumliche Schwerpunkte unterstützt und andererseits die übrigen Gebiete lediglich mit noch zu definierenden Mindeststandards versorgt werden sollen. Dieser Wegdie Förderung von tatsächlichen oder vermeintlichen Wachstumszentren - würde aber die bisherige Förderpolitik auf den Kopf stellen.

Zusammenfassend lässt sich aus der Entwicklung der Vergangenheit kein eindeutiger Beleg für oder gegen die Konvergenzhypothese ableiten, soweit Regionen unterhalb des nationalen Niveaus herangezogen werden. Die meisten vorliegenden Studien belegen eine langsame Konvergenz auf globaler, nationaler Ebene oder zumindest mit Blick auf einige Regionen (Konvergenzclubs) (Eckey/Türk 2006). Das bereits seit mehreren Jahren zu beobachtende Bild zeigt sich auch in einer EU-27: Die wirtschaftlichen Disparitäten zwischen den
EU-Mitgliedsländern verringern sich, die regionalen Unterschiede innerhalb vieler Mitgliedstaaten nehmen zu. Insbesondere in den wachsenden Volkswirtschaften vergrößert sich die Kluft zwischen städtischen Agglomerationen und den ländlichen Räumen (Kramar 2006).

\section{Aktuelle politische Ansätze für einen Konvergenz- prozess in Europa}

Die Europäische Union unternimmt in letzter Zeit erhebliche Anstrengungen, um die Kräfte zu bündeln und die Wachstumspotenziale besser zu nutzen. Diese umfassen Elemente einer Industrie- und Dienstleistungspolitik sowie die Regionalpolitik. Beispiele hierfür werden in dieser Ausgabe der WSI-Mitteilungen dargestellt. Weitere Beispiele sind die Ansätze zu einer gemeinsamen Energiepolitik, die Bündelung der europäischen Luftfahrtindustrie sowie die Chemikalien-Verordnung als Kompromiss zwischen den unterschiedlichen Interessen von Industrie und Beschäftigten, Ökologie und Verbrauchern. Darüber hinaus soll die Forschung forciert werden und mit dem Europäischen Forschungsrat (ERC), für den $15 \%$ des Budgets des 7. Rahmenprogramms vorgesehen sind, soll erstmals Grundlagenforschung in der EU gefördert werden. Der ERC ist jedoch kein Instrument für den Regionalausgleich, er soll Exzellenz fördern. Das geplante European Institute of Technology (EIT) dient dem gleichen Ziel der Exzellenz. Es soll eine Art "Counter Part“ zum Massachusetts Institute of Technology (MIT) werden. Hier gibt es jedoch Widerstände, sodass seine Gründung fraglich ist. Gedacht ist von Seiten der EU-Länder an die Förderung und Vernetzung guter Hochschulen im Sinne einer Exzellenz-Initiative für Europa.

Zwar sieht es im Bereich der Regionalförderung etwas anders aus. Hier liegt mit den Europäischen Strukturfonds schon seit Langem ein konzeptionell einheitlicher Rahmen vor. Aber die nur mäßigen Erfolge der regionalen Wirtschaftspolitik in der EU stellten im Vorfeld der im Sommer 2006 beschlossenen Neukonzeption der Europäischen Strukturfonds die Effizienz und Effektivität des regionalpolitischen Instrumentariums in Europa zunehmend infrage bzw. es wurde eine konzeptionelle Neu- ausrichtung dieses Politikbereiches gefordert. Im Mittelpunkt der Diskussion standen die Fragen, ob das Ziel der europäischen Regionalpolitik in einer Angleichung der Einkommen und der Beschäftigungschancen in den schwachen Regionen bestehen bleiben soll (Ausgleichsziel), oder ob förderwürdige Regionen innerhalb des europäischen Binnenmarktes international wettbewerbsfähig gemacht werden sollen (Wachstumsziel). Bei einer Ausgleichspolitik wären die Regionen förderfähig, die einen politisch festgelegten Schwellenwert bei den Einkommens- und Beschäftigungsbedingungen nicht überschreiten. Im zweiten Fall müsste sich die Regionsauswahl nach wachstumsorientierten Gesichtspunkten an Kriterien der regionalen Wettbewerbsfähigkeit ausrichten. Und die förderwürdigen Regionen müssten bereits über ein ausreichendes Potenzial, unter anderem im Hinblick auf ihre Siedlungsstruktur, ihre Einwohnergröße, ihren Unternehmensbestand, ihre Forschungsund Wissenstruktur, ihre Absatz- und Bezugsmärkte, verfügen. Unterstützung fand die wachstumsorientierte Regionalpolitik durch den Sapir-Bericht, ${ }^{3}$ der bereits im Jahr 2003 zu dem Ergebnis kam, dass die EU trotz beachtlicher institutioneller Errungenschaften in wirtschaftlicher Hinsicht uneinheitlich abschneide. Zwar wurden in den 1990er Jahren die makroökonomische Stabilität erheblich verbessert und der hohe Stellenwert der Kohäsion aufrechterhalten, doch sei es dem EU-System nicht gelungen, ein zufriedenstellendes Wachstum zu erzielen. Die Sapir-Gruppe schlug der Europäischen Kommission deshalb vor, die Europäischen Strukturfonds ab 2007 im Sinne der Lissabon-Strategie unter wachstumsorientierten Aspekten

\footnotetext{
3 Ziel des Berichts der Expertengruppe unter Vorsitz von André Sapir sollte sein, die Auswirkungen der beiden strategischen Wirtschaftsziele zu analysieren, die sich die EU für das 2010 auslaufende Jahrzehnt gesetzt hat: die Union zum wettbewerbsfähigsten und dynamischsten wissensbasierten Wirtschaftsraum mit dauerhaftem Wirtschaftswachstum und größerem sozialen Zusammenhalt zu machen (die sogenannte "Lissabon-Strategie) und die bevorstehende Erweiterung durch eine rasche Hebung des Lebensstandards in den neuen Mitgliedstaaten zum Erfolg zu führen. Die Gruppe sollte das gesamte System der EU-Wirtschaftspolitiken überprüfen und eine Strategie zur Steigerung des Wachstums bei gleichzeitiger Sicherung von Stabilität und Zusammenhalt in einer erweiterten Union vorschlagen (Sapir André Groupe 2003)
} 
einzusetzen und diese auf die Wachstumszentren zu konzentrieren.

Da dieser Ansatz bisher politisch nicht durchzusetzen ist, stellt die europäische Regionalpolitik nach der Reform der Europäischen Strukturfonds einen Kompromiss zwischen beiden Ansätzen dar. Im Einklang mit dem Artikel 158 des EG-Vertrages, der der Gemeinschaft vorschreibt, "eine harmonische Entwicklung der Gemeinschaft als Ganzes” zu fördern und „die Unterschiede im Entwicklungsstand der verschiedenen Regionen und den Rückstand der am stärksten benachteiligten Gebiete oder Inseln, einschließlich der ländlichen Gebiete, zu verringern", baut das System der europäischen Strukturpolitik nach wie vor auf der Solidarität zwischen den strukturstarken und -schwachen Regionen auf. Dieser ausgleichspolitische Ansatz konzentriert die strukturpolitischen Hilfen auf die strukturschwächsten Gebiete Europas. Gleichzeitig wird ganz im Sinne der Lissabon-Strategie ein Teil der Europäischen Strukturfonds unter wachstumsorientierten Aspekten eingesetzt.

Die Europäische Kommission konzentriert die Europäischen Strukturfonds, die mit einem Gesamtbudget in Höhe von 336 Mrd. $€$ ausgestattet sind, im Zeitraum von 2007 bis 2013 auf drei Schwerpunkte: ${ }^{4}$

(1) Das Ziel „Konvergenz“ unterstützt (wie das alte Ziel 1 aus der abgelaufenen Förderperiode) die Regionen in Europa mit dem größten Entwicklungsrückstand. Dazu gehören die Regionen der neuen Mitgliedsländer, aber auch Ostdeutschland und der Regierungsbezirk Lüneburg. Ziel ist die Steigerung und qualitative Verbesserung der Investitionen in physische und Humanressourcen, die Entwicklung der Innovation und der Wissensgesellschaft, die Förderung der Anpassungsfähigkeit an den Wandel in Wirtschaft und Gesellschaft, der Schutz und die Verbesserung der Umwelt. Dieses Ziel bindet die meisten Mittel - über $80 \%$.

(2) Das Ziel „Regionale Wettbewerbsfähigkeit und Beschäftigung" soll in allen anderen Regionen jenseits des Ziels „Konvergenz" verfolgt werden. Inhaltlich stützt es sich auf zwei Pfeiler - einen regionalen und einen horizontalen Ansatz. Der regionale Pfeiler soll den strukturellen Wandel in industriellen, städtischen und ländlichen $\mathrm{Ge}$ bieten fördern und voranbringen. Im Bereich der regionalen Wettbewerbsfähigkeit geht es in erster Linie um die Unterstützung der Lissabon-Strategie und hauptsächlich um die Förderung von Innovation und Wissensgesellschaft. Das neue Ziel 2 wurde in die Verantwortung der Mitgliedstaaten gelegt, die die förderwürdigen Regionen selbst auswählen. Der nationalorientierte Pfeiler, der sich an der europäischen Beschäftigungsstrategie orientiert, soll sich auf Maßnahmen zur Förderung von Vollbeschäftigung, Arbeitsplatzqualität, Arbeitsproduktivität und soziale Integration konzentrieren. Knapp $16 \%$ der Mittel fließen in das Ziel „Regionale Wettbewerbsfähigkeit und Beschäftigung“.

(3) Das Ziel „Europäische territoriale Zusammenarbeit" soll die Erfahrungen aus der Gemeinschaftsinitiative INTERREG fortführen und verfolgt die Stärkung der grenzüberschreitenden Zusammenarbeit in der EU. Dafür stehen ca. 2,5 \% der Mittel zur Verfügung.

\section{Politischer Ausblick}

Wenn Europa seine Potenziale nutzt, dann verringert sich der Widerspruch zwischen der Ausgleichszielsetzung und dem Wachstumsziel. Eine Studie der Hans-BöcklerStiftung hat im Vergleich von 15 in Europa durchgeführten innovationspolitischen Projekten gezeigt, dass eine regionale Innovationspolitik sehr wohl erfolgreich sein kann, wenn sie mit sozial-integrativen Maßnahmen gekoppelt ist. Innovationspolitische Vorhaben sollten deshalb Elemente zur Erhaltung und Schaffung eines polarisationsfreien sozialen Umfeldes in ihren Aufgabenkatalog aufnehmen und die Fähigkeit unter Beweis stellen, Lernprozesse, wie z. B. Qualifizierung, Kooperation und Kommunikation, zu integrieren (Guth et al. 2005). Eine gute Kohäsionspolitik ist somit eine zentrale Voraussetzung für die Stimulierung der Wachstumspotenziale in Europa - wie insbesondere die skandinavischen Länder erfolgreich gezeigt haben. Im Prinzip kann ein solcher Ansatz durch Instrumente der EU, wie der soziale Dialog und die offene Methode der Koordination (Schäfer 2006), gestützt werden.

Die neue Strukturförderung hat das Prinzip der regionsbezogenen Strukturförderung und einer gebietsunabhängigen $\mathrm{Be}$ schäftigungsförderung beibehalten, jedoch hat sie die nationalen Spielräume der Mitgliedstaaten erheblich ausgeweitet. Damit muss die Strukturpolitik einen ganz neuen Spagat bewältigen, während sie gleichzeitig an Einheitlichkeit und Kontrollmöglichkeiten durch die Kommission verliert. Außerdem verfolgt sie mit den beiden regionalen Zielen „Konvergenz" und „Regionale Wettbewerbsfähigkeit" zwei konkurrierende Ziele - nämlich Ausgleich und Wachstum. Ob dieser Ansatz zielführender ist als das bisherige Konzept, bleibt fraglich.

Die Diskussion, die im Vorfeld der reformierten Europäischen Strukturfonds geführt wurde, macht zunehmend deutlich, dass die Frage, welches Ausmaß an Entwicklungsunterschieden zwischen den Mitgliedstaaten und ihren Regionen in einer Wirtschafts- und Sozialunion überhaupt zulässig ist, beantwortet werden muss. Die Antwort ist normativ und hängt von gesamtwirtschaftlichen Rahmenbedingungen und - dies wird in der Diskussion leicht übersehen - von der gesellschaftspolitischen Akzeptanz ab. Die gescheiterte Verfassung sowie die Diskussion um die Dienstleistungsrichtlinie sind nur zwei - aber prominente - Beispiele, die zeigen, dass die EU nur dann erfolgreich ist, wenn sie neben einer Wirtschafts- auch eine entsprechende Sozialunion darstellt, die die Menschen dort abholt, wo sie leben - in den Regionen. Die Europäischen Strukturfonds sind dazu ein wichtiges Instrument. Bei ihrem Einsatz darf aber eines nicht vergessen werden: Angesichts der Erfahrungen mit der Regionalentwicklung in Westdeutschland in der Nachkriegszeit braucht eine Regionalpolitik einen langen Atem, und es wäre eine Illusion, zu glauben, dass die EU nur annähernd die derzeitigen regionalen Divergenzen in der EU-27 mittelfristig ausgleichen könnte.

Trotz mancher positiver Ansätze muss jedoch konstatiert werden, dass in Europa eine neue Ära der Strukturpolitik eingeleitet wurde, die aus beschäftigungspolitischer Sicht ambivalent zu beurteilen ist. Neben die bisherige ausgleichspolitische Orientierung sind dadurch immer mehr wachstumspolitisch motivierte Maßnahmen getreten. Zwar ist dieser Konflikt in der europäischen Strukturpolitik nicht neu

\footnotetext{
4 Die entsprechenden Verordnungstexte sind im Internet abrufbar: http://ec.europa.eu/regional_policy/sources/docoffic/official/regulation/newregl0713_de.htm.
} 
- von Anfang an musste sie einen Spagat zwischen Ausgleich und Wachstum machen -, neu ist allerdings die Dimension, in der die Kritiker einer ausgleichenden Strukturpolitik mittlerweile erfolgreich sind. Denn in vielen Bereichen wird heute Strukturpolitik mit reduzierten Finanzmitteln unter Wachstumsgesichtspunkten betrieben.

Die Ausrichtung der „neuen“ Strukturpolitik rein am fiskalischen Aspekt und nicht an ihren Inhalten löst die derzeitigen strukturellen Probleme in der Wirtschaft und am Arbeitsmarkt kaum. Für die weitere Diskussion bleibt nur zu hoffen, dass die Wirkungsanalysen, die den Wachstumseffekt des bisherigen Systems belegen, stärker beachtet werden.
Gleich welche konzeptionelle Richtung die Wirtschafts- und Strukturpolitik einschlägt, sie muss in erster Linie an der Sicherung und Schaffung von Arbeitsplätzen in ausreichendem Umfang und deren Qualität im Rahmen einer modernen Unternehmens- und Innovationsentwicklung gemessen werden. Der Strukturwandel ist als Chance für eine eigenständige, zukunftsfähige, wirtschaftliche Entwicklung in Europa zu verstehen. Die Vernetzung der europäischen Wirtschaft mit internationalen Wertschöpfungsketten ist für die weitere Wirtschaftsentwicklung genauso notwendig wie die Entwicklung der $\mathrm{Hu}-$ manressourcen vor Ort. Problemlösungen zwischen Betrieb, Branche und Region sind dann Erfolg versprechend, wenn unter- schiedliche Akteure an dem Prozess beteiligt sind und ein differenzierter Einsatz von Förderinstrumenten und -strategien organisiert werden kann. So ist die Zusammenarbeit in Unternehmensclustern dann besonders wirkungsvoll, wenn sie sich nicht nur auf die Anwendung neuer Technologien, sondern auch auf Weiterbildung, Qualifizierung und innovative Arbeitszeitgestaltung bezieht. In den Fokus der Strukturpolitik muss viel stärker als bisher die Förderung von Wertschöpfungsketten, Unternehmensverbünden, regionalen Netzen oder auch Beschäftigungspakten rücken.

\section{LITERATUR}

Belitz, H./Kirn, T./Werwatz, A. (2006): Innovationsfähigkeit: Deutschland braucht mehr Schwung, DIW-Wochenbericht 45, Berlin Bundesministerium für Bildung und Forschung (BMF) (2005): Zur technologischen Leistungsfähigkeit Deutschlands 2005, Bonn/Berlin Eckey, H.-F./Türk, M. (2006): Convergence of EU-Regions. A Literature Report, Volkswirtschaftliche Diskussionsbeiträge 80, Kassel Europäische Kommission (2006): Die Strategie für Wachstum und Beschäftigung und die Reform der europäischen Kohäsionspolitik. Vierter Zwischenericht über den Zusammenhalt, KOM 281, Brüssel European Commission (2006): European Commission Occasional Papers 24, Brüssel

Guth, M./Jakobs, S./Kodré, P./Mühlenfeld, C./Noetzel, R. (2005): Erfolgsdeterminanten für eine sozialintegrative regionale Innovationspolitik - Vergleichende Analyse von 15 Fallbeispielen. Endbericht zu einem Projekt der Hans-Böckler-Stiftung, im Erscheinen
Kramar, H. (2006): Economic convergence on different spatial levels: the conflict between cohesion and growth, in: RuR 1, S. 18-27

Lundvall, B. A. (1992): National Systems of Innovation: Towards a Theory of Innovation and Interactive Learning, London OECD (2004): Main Science and Technology Indicators 2004 Sapir André Group (2003): An agenda for a growing Europe - Making the EU Economic System Deliver, Brüssel Schäfer, A. (2006): Aufstieg und Grenzen der Offenen Methode der Koordinierung, in: WSI-Mitteilungen 10, S. 540-546 Schmidt, N. (2006): Innovation, in: Urban, H.-J. (Hrsg.): ABC zum Neoliberalismus, Hamburg 